\title{
A brief review on the presentation of cycle 24, the first integrated solar cycle in the new millennium
}

\author{
K. J. Li ${ }^{1,2}$, W. Feng ${ }^{3}$, H. F. Liang ${ }^{4}$, L. S. Zhan ${ }^{5}$, and P. X. Gao ${ }^{1}$ \\ ${ }^{1}$ National Astronomical Observatories/Yunnan Observatory, Chinese Academy of Sciences, Kunming 650011, China \\ ${ }^{2}$ Key Laboratory of Solar Activity, National Astronomical Observatories, Chinese Academy of Sciences, Beijing 100012, \\ China \\ ${ }^{3}$ Research Center for Analysis and Measurement, Kunming University of Science and Technology, Kunming 650093, China \\ ${ }^{4}$ Department of Physics, Yunnan Normal University, Kunming 650093, China \\ 5 Jingdezhen Ceramic Institute, Jingdezhen 333001, Jiangxi, China
}

Received: 7 September 2010 - Revised: 28 December 2010 - Accepted: 25 January 2011 - Published: 15 February 2011

\begin{abstract}
The status of the extended solar activity minimum, since the second half of 2007 , has been briefly instructed to the solar-terrestrial community. Cycle 24 has the most spotless days since cycle 16 , and probably even since the modern cycles, latitudes of high-latitude $\left(>35^{\circ}\right)$ sunspots belonging to a new cycle around the minimum time of the cycle are statistically the lowest at present, compared with those of cycle 12 onwards, and there is only one or no sunspots in a month appearing at high latitudes $\left(>20^{\circ}\right)$ for 58 months (from November 2003 to September 2008), which is observed for the first time since cycle 12 onwards. The solar wind velocity and pressure, $10.7 \mathrm{~cm}$ solar radio flux, the polar solar magnetic field, solar total irradiance, and so on reach their minima during the 23-24 cycle minimum time. In order to explain the present extreme low activity, we introduced here one possible mechanism using helio-seismology observations. Viewing, from the long-term running of the time scales of both the Gleissberg period and millenniums, the extended solar activity minimum becomes logical. According to the present observations, the cycle 24 should start in November 2008. Solar activity is predicted at being about $30 \%$ lower in cycle 24 than in cycle 23 , synthesizing the typical predictions of solar activity, including those given by NASA and NOAA. The 24th solar cycle is sluggishly coming and it should be an opportune moment for studying solar physics and solar-terrestrial physics.
\end{abstract}

Keywords. Solar physics, astrophysics, and astronomy (General or miscellaneous)

\section{Correspondence to: $\mathrm{K}$. J. Li}

(lkj@ynao.ac.cn)

\section{Introduction}

It is well known that sunspot activity waxes and wanes within an approximate 11-year Schwabe cycle, and sunspot activity should be reduced to a valley when a solar cycle is progressing into its next new cycle. Thus, people are seemingly not surprised for the present low level of sunspot activity, appearing at the solar minimum time between cycles 23 and 24 . However, when reviewing the history of the detailed observations of sunspots, people should find that the present low level of sunspot activity seems abnormal, since such a low sunspot activity was rarely recorded before. For example, in the year of 2008, the number of spotless days reached 266 days, accounting for $73 \%$ of the total days in the whole year. In the last hundred years, only the year 1913 had even more spotless days: in that year, the number of spotless days reached 311 days, accounting for $85 \%$ of the total days in that whole year. In the first 4 months (120 days) of the year 2009, there were 103 spotless days, accounting for $86 \%$, and in this year, there were 262 spotless days, just 4 days less than those in 2008, and such a year having so many spotless days ranks 3 for the last hundred years ${ }^{1}$. The present Sun is experiencing the first valley of sunspot activity in this century and this new millennium, and the current solar cycle transit has surprisingly accumulated 806 spotless days so far (till August 2010). The current sunspot activity has been at so deep a level for such a long time, some scientists, therefore, speculate that the Sun might entre a long period of no (or very low) sunspot activity, similar to the Maunder minimum in the seventeenth century ${ }^{2}$. It is claimed that there should be a positive correlation between solar activity variations and the

\footnotetext{
${ }^{1}$ http://users.telenet.be/j.janssens/Spotless/Spotless.html

${ }^{2} \mathrm{http} / / /$ solarscience.msfc.nasa.gov/
}

Published by Copernicus Publications on behalf of the European Geosciences Union. 


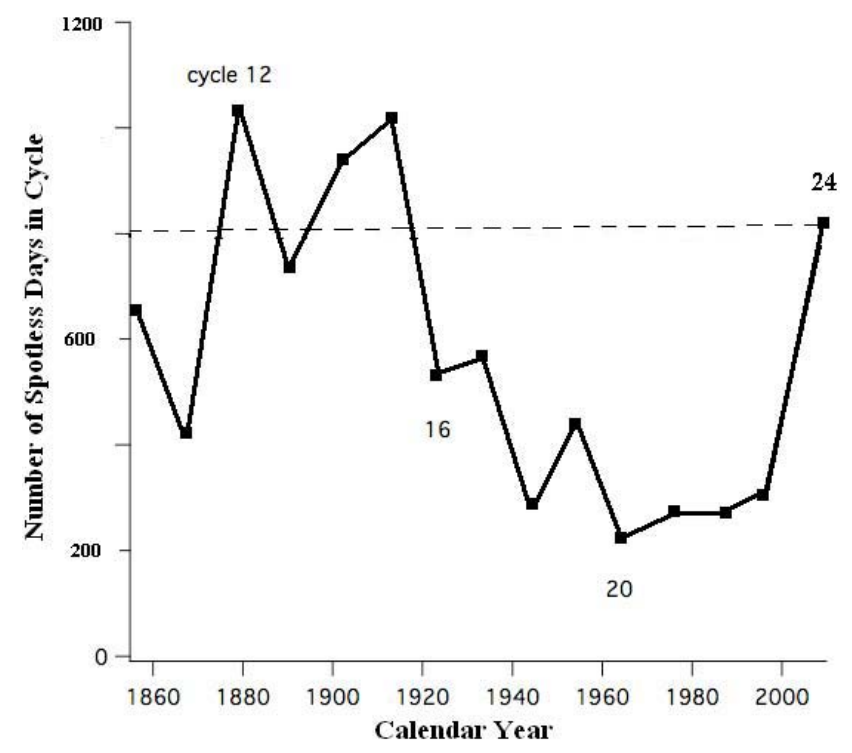

Fig. 1. The number (the filled squares) of spotless days in solar cycles 10 to 24 .

Earth surface temperature (Eddy, 1976; Usoskin, 2008). The Sun went through three stages of great changes of solar activity in the last millennium: the Medieval maximum coinciding with the Medieval Warm Period, the Maunder minimum coinciding with the coldest excursion of the Little Ice Age, and the Sporer minimum coinciding with the other severe temperature dip of the Little Ice Age (Eddy, 1976). It is necessary to investigate the present status and future prospects of solar activity, due to its influence and importance in solar physics and solar-terrestrial physics (Gao et al., 2008; Usoskin, 2008). This paper, mainly as an instruction to the solar-terrestrial community, comprehensively reports the present status of a variety of solar activity indices and the future prospects of solar activity, the interpretation of the present low level of solar activity and the potential opportunity to improve solar physics and solar-terrestrial physics.

\section{The present status of solar activity}

The reliable time series of sunspot number is generally thought to start from solar cycle 10, and it is called the start of the modern solar activity cycles (Wilson et al., 1996; Li et al., 2004). Figure 1 shows the number of spotless days per cycle during the modern solar activity cycles. The data used here are available on the spotless days web site ${ }^{3}$. Up to the end of August 2010, the number of spotless days reached 806 days for cycle 24 . Cycle 24 has the most spotless days from cycle 16 onwards and is only fewer than cycles 12,14 , and 15 among the modern solar activity cycles. The ability of observing sunspots is believed to improve with the elapse of

\footnotetext{
${ }^{3}$ http://users.telenet.be/j.janssens/Spotless/Spotless.html
}

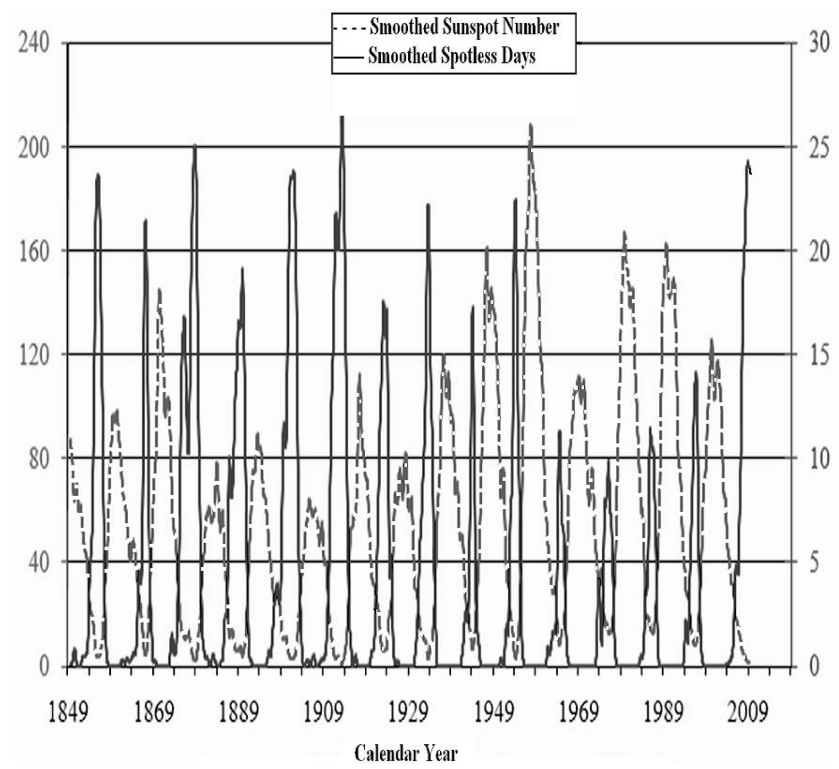

Fig. 2. The smoothed monthly number of spotless days (solid line, right scale) and smoothed monthly sunspot number (dashed line, left scale). The data used here are available from the spotless-day web site (http://users.telenet.be/j.janssens/Spotless/Spotless.html).

time since cycle 10, and Li (2009) inferred that some small sunspots would not be observed during the minimum times of cycles 12 to 15 . Thus, cycle 24 should have the most spotless days, probably even for all the modern solar cycles. Therefore, low sunspot activity is inferred to occur only once in the past one hundred years (Li, 2009). Solar cycle 24 is the first integrated solar cycle in the new millennium and it should progress into the new century and the new millennium with extended low activity.

Figure 2 shows the smoothed monthly number of spotless days (updated to August 2010) and the smoothed monthly sunspot number, and the data used here are available also from the spotless days web site. From the figure, we can find that the smoothed monthly number of spotless days also waxes and wanes within a "cycle", similar to the smoothed monthly sunspot number. Since 1849, 14 integrated cycles have been informed for the two indexes. However, the two indexes are in complete anti-phase with each other. At present, the monthly number of spotless days for cycle 24 just passed its peak and is going into its deceasing part of the cycle. In the future, the total number of spotless days for cycle 24 is expected to increase continuously, so that the monthly number of spotless days for cycle 24 forms its integrated cycle.

The continuous observations and compilations of the position and area of sunspots began at Greenwich Observatory in the year 1874, which recorded the position of sunspots at the solar disk for 12 complete cycles since cycle 12 onwards. Li (2009) found that the mean latitude of high-latitude $\left(>35^{\circ}\right)$ sunspots in cycle 24 is the lowest since cycle 12 onwards 

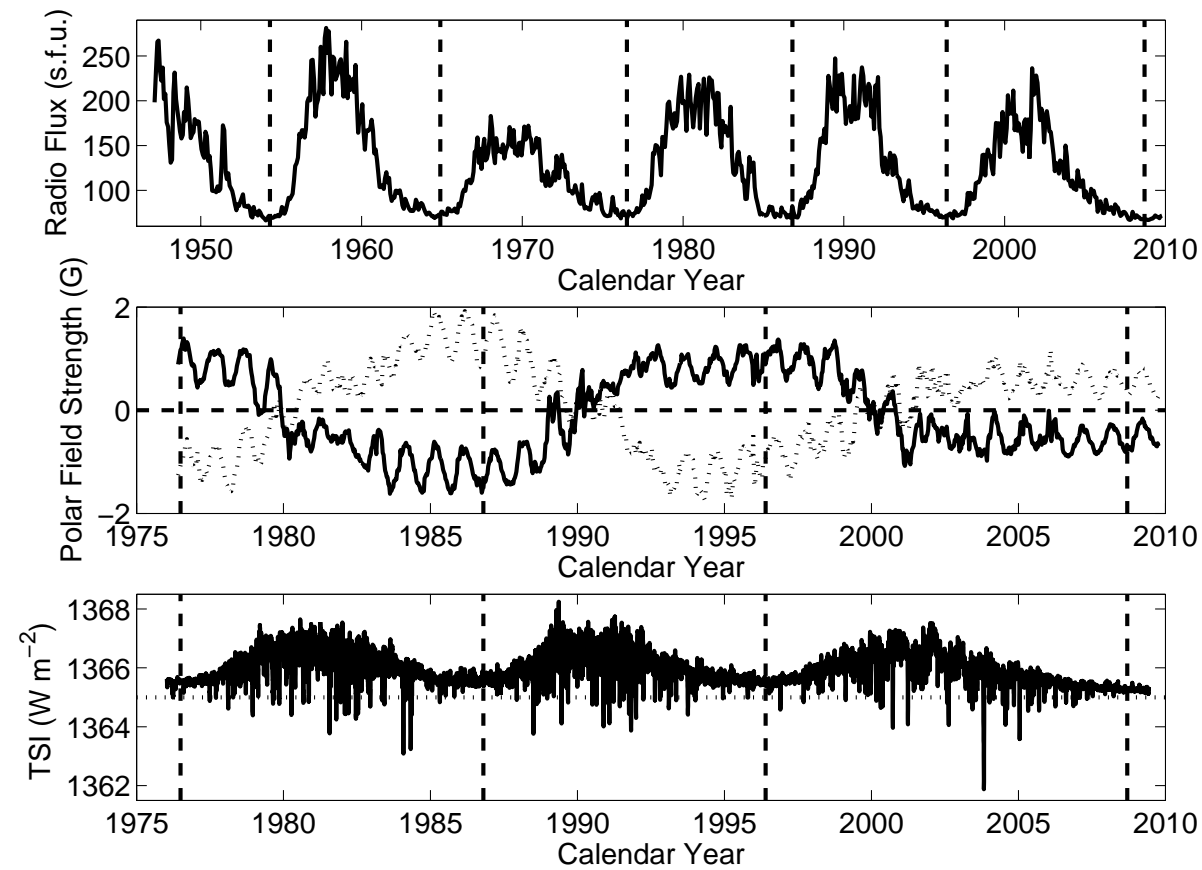

Fig. 3. Top panel: the monthly mean values of the solar radio adjusted flux density at $10 \mathrm{~cm}$ wavelength measured by Ottawa/Pentiction from February 1947 to May 2009. Middle panel: the monthly mean values of the Sun's polar field strength in the Northern (solid line) and Southern (dashed line) Hemispheres, observed at Wilcox Solar Observatory (WSO) from June 1976 to September 2009. Bottom panel: the daily total solar irradiance (TSI) of the PMOD composite from 1 December 1976 to 9 June 2009. The vertical dashed lines mark the minimum times of sunspot cycles.
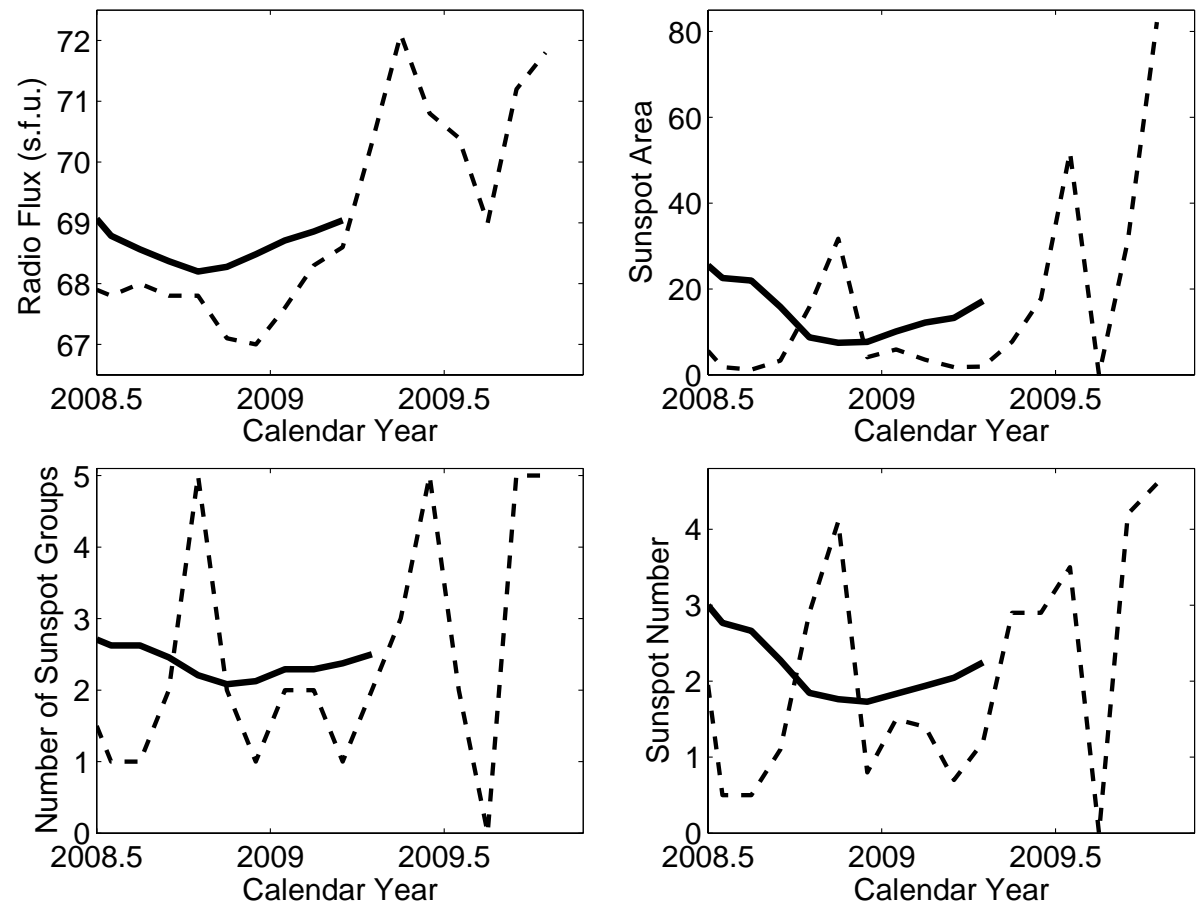

Fig. 4. Top left panel: the monthly mean values of the solar radio adjusted flux density at $10 \mathrm{~cm}$ wavelength measured by Ottawa/Pentiction (dashed line); top right panel: the monthly mean sunspot areas (dashed line); bottom left panel: the monthly number of sunspot groups (dashed line); and bottom right panel: the monthly mean sunspot numbers (dashed line). All the above data span from January 2008 to September 2009. Their corresponding 13-point smoothed values (solid line) are also shown in their corresponding panels. 


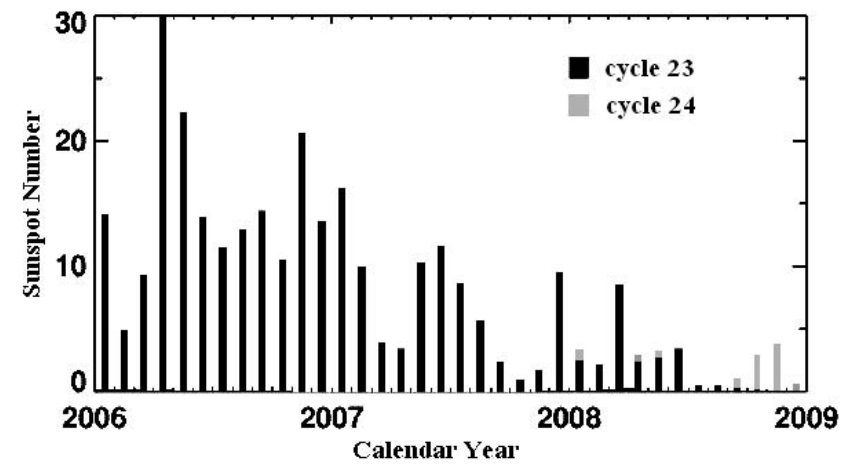

Fig. 5. The monthly number of sunspot groups since the year 2006 onwards for cycles 23 (dark grey) and 24 (light grey), respectively.

(till the end of September 2009, recorded was only one highlatitude $\left(>35^{\circ}\right)$ sunspot, appearing on 23 September 2009). Only one or no sunspots per month appeared at high latitudes $\left(>20^{\circ}\right)$ from November 2003 until September 2008, surprisingly spanning as long as 58 months, such a status is the first time observed since cycle 12 . The maximum amplitude of a solar cycle is found to be positively correlated to the mean latitude of sunspots at high latitude $\left(>35^{\circ}\right)$ over the cycle ( $\mathrm{Li}$ et al., 2003), thus, cycle 24 is expected to present a rather low level of solar activity.

The continuous observations of the solar polar (latitudes are larger than $55^{\circ}$ ) field strength began in the year 1976 . Figure 3 shows the monthly mean values of the Sun's polar field strength in the Northern and Southern Hemispheres, respectively, observed at Wilcox Solar Observatory (WSO) from June 1976 to September 2009. The monthly mean values of the Sun's polar field strength are in complete antiphase with the low-latitude sunspot activity. The monthly mean values of the Sun's polar field strength during the minimum time of a cycle for cycle 24 are weaker than those for the other cycles, being the lowest since the beginning of the observations on the Sun's polar field strength. The solar high-latitude polar field strength averaged over a month at the minimum time of a cycle is found to be positively correlated with the maximum monthly mean low-latitude magnetic field strength of the same cycle (Svalgaard et al., 2005a, b), thus, cycle 24 is expected to have a rather low level of solar activity, even potentially the smallest cycle in the last 100 years, according to the prediction by Svalgaard et al. (2005a, b).

The solar index of $10.7 \mathrm{~cm}$ solar radio flux (F10.7) is a reliable and sensitive activity index, and the continuous observation of solar radio adjusted flux density at $10 \mathrm{~cm}$ wavelength $(2800 \mathrm{MHz})$ began in the year 1947 (Svalgaard and Hudson, 2010, and reference therein). Figure 3 shows the monthly mean values of the solar radio adjusted flux density at $10 \mathrm{~cm}$ wavelength measured by Ottawa/Pentiction from February 1947 to May 2009. The solar radio adjusted flux density at the minimum time of a cycle for cycle 24 is the smallest among all cycles since the beginning of the radio flux density measurement.
The continuous observation of total solar irradiance (the so-called solar constant) began in the year 1976. Figure 3 also shows the daily total solar irradiance (TSI) of the PMOD composite from 1 December 1976 to 9 June 2009 (Frohlich, 2006, 2009). TSI at the minimum time of cycle 24 is the lowest since the beginning of TSI measurement.

Other solar activity indices, such as low-speed solar wind speed, solar wind pressure, photospheric magnetic open fields, are all the lowest since the beginning of their measurement ${ }^{4}$.

\section{The determination of the start time of solar cycle 24}

The Hale cycle, in which the magnetic polarity of sunspot pairs (preceding and following sunspots) reverses between alternate cycles. The sunspot in AR 10981, having opposite polarities from the previous cycle, emerged at high latitude $\left(30^{\circ}\right)$ on 4 January 2008 and disappeared on 6 January 2008, suggesting that the extended cycle 24 should begin in January 2008 in high-latitude regions ${ }^{5}$. Helioseismic observation indicates that, in the solar interior, the solar activity cycle 24 started in late 2007 (Salabert et al., 2009), consistent with the above result. Thus, it is referred that the extended cycle 24 should begin around the beginning of 2008.

We use the Royal Greenwich Observatory dataset to count the monthly numbers of sunspot groups from January 2008 to September 2009, which are shown in Fig. 4. The figure also shows the monthly mean values of the solar radio adjusted flux density at $10 \mathrm{~cm}$ wavelength measured by Ottawa/Pentiction, the monthly mean sunspot numbers and the monthly mean sunspot areas during the same time interval. Also shown in the figure are their corresponding 13point smoothed values. The smoothed monthly mean values of the solar radio adjusted flux density at $10 \mathrm{~cm}$ wavelength reach its minimum in October 2008; the smoothed monthly mean sunspot numbers reach its minimum in December 2008, whose value is 1.7 , being the second smallest minimum value of a cycle since cycle 10 onwards (the lowest is 1.5 which occurred in cycle 15); the smoothed monthly numbers of sunspot groups and the smoothed monthly mean sunspot areas both reach their minima in November 2008.

The time, when the number of high-latitude sunspot groups of a new solar cycle first exceeds the number of lowlatitude sunspot groups of the previous solar cycle, is also used to mark the start of the new solar cycle. Figure 5 shows the monthly number of sunspot groups since 2006 onwards for cycles 23 and 24, respectively. From the figure, we can find that the monthly number of sunspot groups for cycle 24 first exceeds that for cycle 23 in September 2008.

To determine the minimum time of solar activity, the above solar activity indices should be considered together (Wilson

\footnotetext{
${ }^{4}$ http://ihy2007.org/IAUWG/WEBPAGES/WG_overview.shtml

${ }^{5} \mathrm{http} / / /$ solarscience.msfc.nasa.gov/
} 
et al., 1996; Harvey and White, 1999). Based on an overall consideration of the above solar activity indices, we determine that cycle 24 should begin in November 2008. The length of cycle 23 then should be 12.5 years, and its fall time should be 8.7 years. Such a cycle length is the longest cycle length, and such a fall time is the longest fall time for observations of the Sun since the start of the modern solar activity cycles.

Cycle 24 is sluggishly coming.

\section{Predictions of the maximum solar activity for solar cycle 24}

Classical solar activity forecasting methods are as follows.

\subsection{Statistical methods based on sunspot cycle characteristics}

The length of a cycle $(L(n)$ for cycle $n)$ is a good indicator of the maximum amplitude of the solar activity in the next cycle $\left(R_{\mathrm{M}}(n+1)\right)$, and there is a significant negative correlation between the two (Hathaway et al., 2002; Watari, 2008; Li, 2009): $R_{\mathrm{M}}(n+1)=-22.91 L(n)+366.58 \pm 23.7$. Thus, $R_{\mathrm{M}}$ should be $75.6 \pm 23.7$ for cycle 24 , based on the above analysis. There is a significant positive correlation between the minimum $\left(R_{\mathrm{m}}\right)$ and maximum $\left(R_{\mathrm{M}}\right)$ amplitudes of the smoothed monthly mean sunspot number of a cycle (Watari, 2008; $\mathrm{Li}, 2009)$. When the data in solar cycles 1 to 23 are used, we have $R_{\mathrm{M}}=78.0+6.0 \times R_{\mathrm{m}} \pm 32.0$. The minimum smoothed monthly mean sunspot number is about 1.7 for cycle 24 , thus, $R_{\mathrm{M}}$ should be $88.2 \pm 32.0$ for cycle 24 . Similar conclusions were obtained using other statistical methods (Du, 2006; Du and Du, 2006; Wang, 2009; Wang et al., 2009).

\subsection{Geomagnetic precursor methods}

Feynman (1982) noted that geomagnetic activity has two different sources: one due to solar activity that follows the sunspot cycle and another due to recurrent high speed solar wind streams that peak during the decline of each cycle. She separated the two by finding the sunspot number dependence of the base level of geomagnetic activity and removing it to reveal the "interplanetary" component of geomagnetic activity (Hathaway and Wilson, 2006). The peaks in the interplanetary component occur prior to the sunspot cycle minimum and are very good indicators for the amplitude of the following sunspot cycle (Hathaway, 2009). Feynman's method proposed that $R_{\mathrm{M}}$ for cycle 24 should be $154 \pm 25$ based on the $a a$ geomagnetic index (Hathaway, 2009).

Thompson (1993) noted that some geomagnetic activity during the previous cycle served as a predictor for the amplitude of the following cycle but, instead of trying to separate the two, he simply related the geomagnetic activity during one cycle to the sum of the amplitudes of that cycle and the following cycle (Hathaway, 2009). Thompson's method proposed that $R_{\mathrm{M}}$ for cycle 24 should be $115 \pm 27$ based on the counting of geomagnetically disturbed days (Hathaway, 2009).

\subsection{Polar field precursor method}

Schatten et al. (1978) suggested using the strength of the Sun's polar field as a predictor for the amplitude of the following cycle based on the Babcock (1961) dynamo model (Hathaway, 2009). $R_{\mathrm{M}}$ for cycle 24 should be $75 \pm 8$ according to the solar polar field precursor method (Svalgaard et al., 2005a, b).

\subsection{Solar dynamo method}

Dikpati and Gilman (2006) predicted that $R_{\mathrm{M}}$ for cycle 24 should be $165 \pm 15$, utilizing a flux-transport dynamo model of solar cycles. The model included a rotation profile and a near surface meridional flow based on helioseismic observations.

Choudhuri et al. (2007) and Jiang et al. (2007) predicted that $R_{\mathrm{M}}$ for cycle 24 should be about 75 , using a similar fluxtransport dynamo but with the surface poloidal field at a minimum as the assimilated data.

The National Aeronautic and Space Administration (NASA) gives its prediction for the monthly mean sunspot numbers of cycle 24 on its web side ${ }^{6}$, so does the National Oceanic and Atmospheric Administration (NOAA) on its web side ${ }^{7}$. NASA believes that the monthly sunspot number of cycle 24 should reach its maximum, about 60 in the year 2013, and NOAA predicts that the maximum monthly sunspot number should be about 90 , occurring in the year 2013. We find that NASA continuously reduced its prediction in the recent years, also indicating that NASA believes in a low level of solar activity in cycle 24 .

In conclusion, it is expected that cycle 24 should be about $40 \%$ weaker than cycle 23 .

\section{The interpretation of a rather low level of solar activity in cycle 24}

Gleissberg (1971) investigated the feature of the maximum smoothed monthly mean sunspot numbers of the solar cycles and found a long-periodic fluctuation in them, about a century in length, called the Gleissberg cycle or century cycle. Figure 6 shows the annual mean sunspot number and their smoothed values from the years 1700 to 2009 (up to 310 year). From the figure, we can find that cycle 24 should be at the valley of a Gleissberg century cycle, which may address the issue why solar activity is so low in cycle 24 .

\footnotetext{
${ }^{6} \mathrm{http}: / /$ solarscience.msfc.nasa.gov/SunspotCycle.shtml

${ }^{7} \mathrm{http}$ ://www.swpc.noaa.gov/SolarCycle/
} 


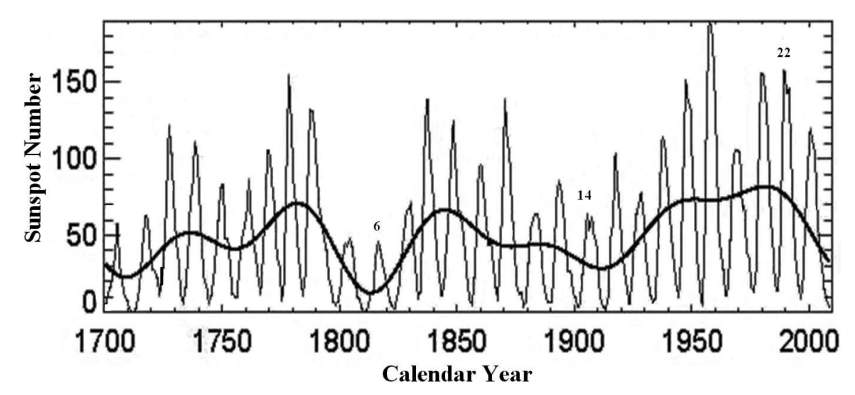

Fig. 6. The annual mean sunspot numbers (thin solid line) and their smoothed values (thick solid line) from 1700 to 2009.

The migrating zonal flow pattern, known as the torsional oscillation, was first detected by Howard and Labonte (1980) in Doppler measurements at the solar surface carried out at the Mount Wilson Observatory; it consists of belts of slightly faster than average rotation that migrate from mid-latitudes to both the equator and poles. The migration of the zonal flow bands during the solar cycle is closely connected to the migration of the magnetic activity belt (Howe et al., 2009; $\mathrm{Li}$ et al., 2008). When the zonal flow bands, marching toward the equator, reaches the critical latitude of 22 degrees, sunspots of the new cycle begin to appear over the solar disk. The zonal flow associated with solar cycle 24 has moved sluggishly, taking three years to migrate 10 degrees in latitudes. However, it takes only two years for solar cycle 23. The present extended solar minimum phase may have resulted from the slower migration of zonal flow bands. The zonal flow bands are now just reaching the critical latitude for cycle 24 , heralding the start of a new solar cycle at the solar disk in the next few months or years. Thus, the recent helioseismic observation result (Howe et al., 2009) does not support that, in cycle 24, the Sun might display the status of no or very few sunspots for a long time, similar to the Maunder minimum in the seventeenth century. Instead, it heralds that the Sun's internal magnetic dynamo continues to operate and sunspot activity for a new cycle already began at the solar disk, low but no stopping ${ }^{8}$.

Solar activity variation is unstable and uneven, undergoing a lot of grand minima and grand maxima, and in the past 11000 years, the Sun spends about $3 / 4$ of the time locating at moderate magnetic-activity level, about $1 / 6$ in grand minima, and about 1/5-1/10 in grand maxima (Usoskin, 2008). The present solar activity is locating at a grand maximum - called the modern grand maximum of solar activity - beginning in the 1920s and lasting about 90 years until now. A total of 19 grand maxima have been identified and most of these grand maxima (about $75 \%$ ) are not longer than 50 years. This suggests that the probability should be rather low for the Sun to still locate in the modern grand maxi-

\footnotetext{
${ }^{8} \mathrm{http}: / /$ science.nasa.gov/science-news/science-at-nasa/2009/ 17 jun_jetstream/
}

mum in the future (Usoskin, 2008). In the future, the Sun should, therefore, present moderate or low magnetic-activity levels. It happened before when solar activity suddenly dropping from a great maximum into a great minimum, for example, the Maunder minimum displays in such a case. It is not surprising even if cycle 24 locates in a great minimum.

\section{Conclusions and discussions}

The level of solar activity is very low around the present solar minimum between cycles 23 and 24: cycle 24 has the most spotless days from cycle 16 onwards and even probably for all of the modern solar cycles; the latitude of highlatitude $\left(>35^{\circ}\right)$ sunspots is the lowest since cycle 12 onwards; only one or no sunspots per month appeared at high latitudes $\left(>20^{\circ}\right)$ from November 2003 until September 2008, surprisingly spanning as long as 58 months, such a status is observed for the first time since cycle 12. From the latter half of 2007, no sunspot or just some low-latitude small sunspots or pores appeared at the solar disk until now. At present, lowspeed solar wind speed, solar wind pressure, $10 \mathrm{~cm}$ solar radio flux, solar polar field strength and total solar irradiance all present their lowest values since the beginning of their measurements. Similar investigations, but of other solar activity indices and geomagnetic indices in cycle 24 are available at the web site 9 and in the reference (Gibson et al., 2009). The extended solar minimum phase may be interpreted by helioseismic observations: it is due to the rather slow migration of the zonal flow bands to the equator for cycle $24^{10}$. Viewing from Gleissberg cycles, cycle 24 is at the valley of a Gleissberg cycle, thus, solar activity is low for cycle 24 . With the aspect of secular cycles, the probability should be rather low for the Sun to still be located in the modern grand maximum in the future, and the Sun may spend its time at moderate or low magnetic-activity levels in the near future.

Based on the monthly mean values of the solar radio adjusted flux density at $10 \mathrm{~cm}$ wavelength, the monthly number of sunspot groups, the monthly mean sunspot areas, the monthly mean sunspot numbers from January 2008 to September 2009 and the moment that the number of highlatitude sunspot groups of a new solar cycle exceeds the number of low-latitude sunspot groups of the previous solar cycle, we determine that cycle 24 should begin in November 2008. The length of cycle 23 should, thus, be 12.5 years, and its fall time should be 8.7 years. Such a cycle length is the longest cycle length, and such a fall time is the longest fall time for observations of the Sun since the start of modern solar activity cycles.

Based on the solar cycle predictions using a variety of classical solar activity forecasting methods in published papers,

\footnotetext{
${ }^{9} \mathrm{http}: / / \mathrm{www}$. solarcycle24.com/

${ }^{10} \mathrm{http}: / /$ science.nasa.gov/science-news/science-at-nasa/2009/ 17 jun_jetstream/
} 
such as statistical methods based on sunspot cycle characteristics, geomagnetic precursor methods, polar field precursor method and solar dynamo method, and the classical solar cycle 24 predictions by NASA and NOAA, it is believed that cycle 24 should be about $40 \%$ weaker than cycle 23 . As far as the accuracy of predictions is concerned, precursor methods are theoretically superior to the statistical methods, because precursor methods are associated with the relationship between solar activity and the used precursor factors ( $\mathrm{Li}$ and $\mathrm{Li}, 2007)$. Predictions for cycles 21 and 22 of strong solar activity indicate that precursor methods are always superior to other prediction methods. However, the error of predictions for weak solar cycle 23 is very large by precursor methods. Solar activity may display the dynamical properties of a low-dimensional chaos (Li et al., 2001). At present, how to accurately predict long-term solar activity is still an open problem.

Solar cycle 24 will give us a great opportunity to further study solar physics and solar-terrestrial physics. The variations in total solar irradiance (TSI) are correlated positively with variations of the troposphere temperature and the seasurface temperature (Eddy, 1976; Usoskin, 2008). The peculiar solar cycle 24 is helpful for the study of the relationship between global warming and variation of solar activity, namely the contribution of variation in TSI to global warming. In the winter of 2009, the Northern Hemisphere cold weather corresponds with the low level of TSI in the year. We expected that the relationship should be clearly defined based on observations of solar activity and global temperature in the future.

The predictions based on the flux-transport dynamo model and the high-diffusivity dynamo model contradict each other. Should solar activity for cycle 24 be $40 \%$ stronger or weaker than for cycle 23? This issue will be addressed in the near future. Helioseismology interpretation for the low level of solar activity in cycle 24 does not resolve the basic questions. The zonal flow band observed by Helioseismology and the magnetic field at the solar surface have the similar cyclic behaviour of solar full-disk activity, and the two are probably only the shadows of a more potent phenomenon occurring within the convection zone (Snodgrass, 1987; Li et al., 2008). How do the zonal flow bands trigger sunspot production, and how do the zonal flow bands emerge? Although helioseismic observation can show the migration of the zonal flow bands (Basu and Antia, 2003; Schou, 2003; Howe et al., 2005, 2006; Antia et al., 2008), these problems are expected to be resolved in the future ${ }^{11}$.

The probability of the Sun entering a new Maunder minimum is rather low at the present time. The Sun's internal magnetic dynamo continues to operate and solar activity should continue to emerge, but the occurrence rate of solar activity should be low. Based on the recent solar observa-

\footnotetext{
${ }^{11} \mathrm{http} / / /$ science.nasa.gov/science-news/science-at-nasa/2009/ 17 jun_jetstream/
}

tions, the sunspots in NOAA AR 1029 emerged on the solar disk on 23 October 2009 and developed, having the largest area in cycle 24 up to now, resulting in a series of flares. In particular, two large X-ray flares were continuously observed on 19 January 2010 in NOAA AR1041 (one flare, a M2.3 flare started at 13:03 UT and peaked at 13:41 UT, ending at 13:50 UT; the other, a M1.7 flare started at 20:23 UT, peaked at 20:35 UT, and ended at 20:46 UT). Thus, observations tell that the Sun is still active, but its activity should be rather low, compared with cycle 23.

The first integrated solar cycle in the new millennium, cycle 24 is sluggishly coming.

Acknowledgements. We thank L. Svalgaard, B. Schmieder and another referee very much for their careful reading of the manuscript and constructive comments, which improved the original version of the manuscript. Data used here are all downloaded from web sites. The authors would like to express their deep thanks to the staff of these web sites. The work is supported by the NSFC under Grants 10873032, 10921303, 11073010, and 40636031, the National Key Research Science Foundation (2011CB811400), and the Chinese Academy of Sciences.

Topical Editor R. Forsyth thanks L. Svalgaard and B. Schmieder for their help in evaluating this paper.

\section{References}

Antia, H. M., Basu, S., and Chitre, S. M.: Solar Rotation Rate and Its Gradients During Cycle 23, ApJ, 681, 680-692, 2008.

Babcock, H. W.: The topology of the Sun's magnetic field and the 22-year cycle, Astrophys. J., 133, 572-587, 1961.

Basu, S. and Antia, H. M.: Changes in Solar Dynamics from 1995 to 2002, ApJ, 585, 553-565, 2003.

Choudhuri, A. R., Chatterjee, P., and Jian, J.: Predicting Solar Cycle 24 With a Solar Dynamo Model, Phys. Rev. Lett., 98, 131103, doi:10.1103/PhysRevLett.98.131103, 2007.

Dikpati, M. and Gilman, P. A.: Simulating and Predicting Solar Cycles Using a Flux-Transport Dynamo, The Astrophysical Journal, 649, 498-514, 2006.

Du, Z. L.: Relationship between solar maximum amplitude and Max-Max cycle length, Astron. J., 132, 1485-1489, 2006.

Du. Z. L. and Du, S. Y.: The relationship between the amplitude and descending time of a solar activity cycle, Solar Phys., 238, 431-437, 2006.

Eddy, J. A.: The Maunder Minimum, Science, 192, 1189-1202, 1976.

Feynman, J.: Geomagnetic and solar wind cycles, 1900-1975, J. Geophys. Res., 87(A8), 6153-6162, 1982.

Frohlich, C.: Solar Irradiance Variability Since 1978, Revision of the PMOD Composite during Solar Cycle 21, Space Sci. Rev., 125, 53-65, 2006.

Frohlich, C.: Evidence of a long-term trend in total solar irradiance. Astron. Astrophys., 501, L27-L30, 2009.

Gao, P. X., Li, K. J., and Li, Q. X.: Latitude migration of solar activity at high latitudes, Chinese Science Bulletin, 53, 8-11, 2008.

Gibson, S. E., Kozyra, J. U., de Toma, G., Emery, B. A., Onsager, T., and Thompson, B. J.: If the Sun is so quiet, why is the Earth ringing? A comparison of two solar minimum intervals, J. 
Geophys. Res., 114(A9), A09105, doi:10.1029/2009JA014342, 2009.

Gleissberg, W.: The Probable Behaviour of Sunspot Cycle 21, Solar Phys., 21, 240-245, 1971.

Harvey, K. L. and White, O. R.: What is solar cycle minimum?, J. Geophys. Res., 104(A9), 19759-19764, 1999.

Hathaway, D. H.: Solar Cycle Forecasting, Space Sci. Rev., 144, 401-412, 2009.

Hathaway, D. H. and Wilson, R. M.: Geomagnetic activity indicates large amplitude for sunspot cycle 24, Geophys. Res. Lett., 33, L18101, doi:10.1029/2006GL027053, 2006.

Hathaway, D. H., Wilson, R. M., and Reichmann, E. J.: Group Sunspot Numbers: Sunspot Cycle Characteristics, Solar Phys., 211, 357-370, 2002.

Howard, R. and Labonte, B. J.: The sun is observed to be a torsional oscillator with a period of 11 years, ApJ, 239, L33-L36, 1980.

Howe, R., Christensen-Dalsgaard, J., Hill, F., Komm, R., Schou, J., and Thompson, M. J.: Solar Convection-Zone Dynamics, 19952004, ApJ, 634, 1405-1415, 2005.

Howe, R., Komm, R., Hill, F., Ulrich, R., Haber, D. A., Hindman, B. W., Schou, J., and Thompson, M. J.: Large-Scale Zonal Flows Near the Solar Surface, Solar Phys., 235, 1-15, 2006.

Howe, R., Christensen-Dalsgaard, J., Hill, F., Komm, R., Schou, J., and Thompson, M. J.: A Note on the Torsional Oscillation at Solar Minimum, The Astrophys. J. Lett., 701, L87-L90, 2009.

Jiang, J., Chatterjee, P., and Choudhuri, A. R.: Solar activity forecast with a dynamo model, Mon. Not. Royal Astronomical Society, 381, 1527-1542, 2007.

Li, K. J.: What does the Sun tell and hint now?, Res. Astron. Astrophys., 9, 959-965, 2009.

Li, Q. X. and Li, K. J.: Low Dimensional Chaos from the Group Sunspot Numbers, Chinese Journal of Astronomy and Astrophysics, 7, 435-440, 2007.

Li, K. J., Yun, H. S., and Gu, X. M.: On long-term predictions of the maximum sunspot numbers of solar cycles 21 to 23, Astron. Astrophys., 368, 285-291, 2001.

Li, K. J., Wang, J. X., Zhan, L. S., Yun, H. S., Liang, H. F., Zhao, H. J., and Gu, X. M.: On the Latitudinal Distribution of Sunspot Groups over a Solar Cycle, Solar Phys., 215, 99-109, 2003.

Li, K. J., Su, T. W., and Liang, H. F.: Periodicity of sunspot activity in the modern solar cycles, Chinese Science Bulletin, 49, 2247$2252,2004$.
Li, K. J., Li, Q. X., Gao, P. X., and Shi, X. J.: Cyclic behavior of solar full-disk activity, J. Geophys. Res., 113, A11108, doi:10.1029/2007JA012846, 2008.

Salabert, D., Garcia, R. A., Palle, P. L., and Jimenez-Reyes, S. J.: The onset of solar cycle 24: What global acoustic modes are telling us, Astron. Astrophys., 504, L1-L4, 2009.

Schatten, K. H., Scherrer, P. H., Svalgaard, L., and Wilcox, J. M.: Using dynamo theory to predict the sunspot number during solar cycle 21, Geophys. Res. Lett., 5(5), 411-414, 1978.

Schou, J.: Helioseismic data analysis, in Stellar Astrophysical Fluid Dynamics, edited by: Thompson, M. J. and ChristensenDalsgaard, J., Cambridge: Cambridge Univ. Press, 247-262, 2003.

Snodgrass, H. B.: Torsional oscillations and the solar cycle, Solar Phys., 110, 35-49, 1987.

Svalgaard, L. and Hudson, H. S.: The Solar Microwave Flux and the Sunspot Number, ASP Conference Series, 428, 325-328, 2010.

Svalgaard, L., Cliver, E. W., and Kamide, Y.: Sunspot cycle 24: Smallest cycle in 100 years?, ASP Conference Series, 346, 401408, 2005a.

Svalgaard, L., Cliver, E. W., and Kamide, Y.: Sunspot cycle 24: Smallest cycle in 100 years?, Geophys. Res. Lett., 32, L01104, doi:10.1029/2004GL021664, 2005b.

Thompson, R. J.: A technique for predicting the amplitude of the solar cycle, Solar Phys., 148, 383-388, 1993.

Usoskin, I. G.: A History of Solar Activity over Millennia, Living Reviews in Solar Physics, 5, 3-86, 2008.

Wang, J. R.: Will the solar cycle 24 be a low one?, Chinese Science Bulletin, 54, 3664-3669, 2009.

Wang, J. L., Zong, W. G., Le, G. M., Zhao, H. J., Tang, Y. Q., and Zhang, Y.: Predicting the start and maximum amplitude of solar cycle 24 using similar phases and a cycle grouping, Res. Astron. Astrophys., 9, 133-136, 2009.

Watari, S.: Forecasting Solar Cycle 24 using the relationship between cycle length and maximum sunspot number, Space Weather, 6, S12003, doi:10.1029/2008SW000397, 2008.

Wilson, R. M., Hathaway, D. H., and Reichmann, E. J.: On the behavior of the sunspot cycle near minimum, J. Geophys. Res. 101, 19967-19972, 1996. 